\title{
Análisis de los procesos de implementación del modelo 1:1 en aulas de Secundaria
}

\author{
Analysis of the implementation process model 1: 1 in secondary \\ classrooms
}

\author{
María José Sosa Díaz ${ }^{1}$, María Rosa Fernández Sánchez ${ }^{2}$ \\ ${ }^{1}$ Facultad de Formación del Profesorado, Universidad de Extremadura, España (mjosesosa@unex.es) \\ ${ }^{2}$ Facultad de Formación del Profesorado, Universidad de Extremadura, España (fersan@ unex.es)
}

\section{RESUMEN:}

En España, el Proyecto Escuela 2.0 ha incorporado el modelo 1:1 en los centros educativos de secundaria. Sin embargo, en la mayoría de los centros escolares no se percibe una plena integración de las TIC en las aulas y, por tanto, su uso normalizado en el proceso de enseñanzaaprendizaje.

En el presente trabajo se pretende realizar una descripción y análisis del tipo de prácticas educativas que se están realizando dentro del modelo 1:1 en el contexto de tres Institutos de Educación Secundaria Obligatoria, para profundizar sobre el estado actual de los procesos de integración de las TIC.

A través de la observación de aula y el análisis de las categorías registradas se puede observar que las prácticas educativas reproducen un modelo tradicional de la enseñanza y no pueden considerarse como experiencias innovadoras. De forma generalizada, las prácticas desarrolladas son individualistas y activas por parte del alumnado. Están basadas en ejercicios pre-elaborados de ensayo error y de corrección inmediata y que no favorece la colaboración entre el alumnado. Sin embargo, se puede afirmar que el uso del ordenador portátil favorece la motivación del estudiante hacia el aprendizaje, además de promover el cambio de rol docente a uno más de orientador y guía. No obstante, la organización y distribución del aula se ha visto influida de manera negativa por la introducción de los ordenadores portátiles en el aula, puesto que obliga a inmovilizar los mesas y establecer modelos tradicionales de organización.

\begin{tabular}{lcr} 
PALABRAS & CLAVE: & \multicolumn{2}{c}{ INNOVACIÓN } \\
EDUCATIVA, & TECNOLOGIA & DE LA \\
INFORMACIÓN & Y COMUNICACIÓN, \\
MODELO 1:1, & EDUCACIÓN & SECUNDARIA \\
OBLIGATORIA. & &
\end{tabular}

\section{ABSTRACT:}

In Spain, the "Project School 2.0" has joined the model 1:1 in Secondary Education Centers. However, in most schools is not perceived the full integration of ITC in classrooms, therefore, its regular use in the teaching-learning process.

This paper aims to provide a description and analysis of educational practices that has been doing inside the 1:1 model in the context of three secondary schools, to deepen about current state of ICT integration processes.

Through classroom observation and analysis of the recorded categories, can be seen that educational practices reproduce traditional teaching models and can't be considered as innovative experiences. Across the board, the developed practices are individualistic and active by students. They are based on different exercises that do not promote 
collaboration among students. However, we can say that laptop promotes students motivation toward learning, and promote teacher's roles change to a counselor and guide role. However, the classroom organization has been influenced negatively by the introduction of laptops, the laptops forces to immobilize the tables and establish traditional models of organization.

KEYWORDS: EDUCATIONAL INNOVATION, INFORMATION TECHNOLOGY AND COMMUNICATION, MODEL 1:1, SECONDARY EDUCATION.

\section{FUNDAMENTACIÓN TEÓRICA}

\subsection{Política de integración de las TIC: Escuela 2.0}

En la última década, en España todas las Administraciones Educativas de cada comunidad autónoma, aunque cada una ha tenido un ritmo diferente, se han propuesto llevar a cabo políticas educativas que tenían como objetivo prioritario favorecer la modernización y la adaptación a la sociedad del conocimiento del sistema educativo a través de medidas de dotación de infraestructuras y equipamiento informático, formación de profesorado y creación de materiales curriculares.

En 2008, para equilibrar el proceso de integración de las Tecnologías de la Información y la Comunicación en todas las Comunidades, a nivel nacional se llevo a cabo el Proyecto de Escuela 2.0 con el objetivo de mejorar la dotación tecnológica en el sistema educativo español que hasta ahora se había ido realizando (Area et al., 2014). Así pues, se manifiesta la importancia de dotar de ordenadores portátiles al alumnado, como el primer eslabón para poner en marcha lo que se ha dado en llamar las aulas digitales del siglo XXI.

El Proyecto Escuela 2.0 se articulará en cinco ejes (Pérez, 2011):

a) Dotación de aulas digitales: de pizarras digitales y de un ordenador portátil propio para cada alumno y profesor, que tendrán a disposición como herramienta de trabajo tanto en clase como en casa.

b) Desarrollo de la conectividad a Internet desde el centro educativo y desde los hogares e interconectividad dentro del aula.

c) Formación del profesorado

d) Generación y acceso a contenidos educativos digitales de carácter curriculares

e) Implicación del alumnado y sus familias en el uso y aprovechamiento de estos recursos.
De este modo, España se incorporó al desarrollo de políticas basadas en el modelo 1:1 o "un ordenador por niño" que ya otros países iberoamericanos como el "Plan CIBAL" en Uruguay, "Un laptop por niño" en Perú, "Conectar Igualdad" en Argentina, o "e-Escolinha" en Portugal, estaban implementando. Se ha producido así un importante avance en la universalización del acceso a las TIC en educación pública con un fuerte componente de equidad social y disminución de la brecha digital (Lagos, 2011; Vaillant, Rodríguez, y Bernasconi, 2015; Valiente, 2011).

Los resultados de algunas investigaciones consultadas revelan que la implementación del Proyecto Escuela 2.0 ha favorecido cambios en la práctica docente (L. Fernández, Correa, y OchoaAizpurua, 2013), aunque la percepción de los profesores es que ha cambiado poco o nada sus prácticas pedagógicas (L. Fernández et al., 2013; Vaillant et al., 2015; Valiente, 2011). No obstante, otras investigaciones consideran que la implantación del Proyecto Escuela 2.0 en el contexto educativo investigado no ha tenido todavía los efectos didácticos deseados en el modo de organizar y gestionar los procesos de enseñanza-aprendizaje (Campión, Navaridas, y Repáraz, 2014; L. Fernández, Losada, y Correa, 2014).

Otros estudios más críticos con la Escuela 2.0 señalan que el proyecto tan solo ha tenido el objetivo de repartir tecnología por los centros y crear infraestructuras, sin dar opción a que cada centro y profesorado decidan como sacar un mejor aprovechamiento didáctico de esas herramientas adaptándolas a su realidad y promover así proyectos de innovación (Murillo, 2010). Por lo que constata que en ningún caso se han producido mejoras significativas en el sistema educativo respecto a la utilización de las TIC.

En este sentido, Area (2011) afirma que una política del modelo 1:1 no puede caracterizarse o entenderse exclusivamente como la mera dotación de computadoras. La presencia y disponibilidad de mucha tecnología en las aulas es una condición necesaria, pero insuficiente para la mejora y el cambio educativo. Este modelo debe ir acompañado de un adecuada planificación de la cultura organizativa y dirección de los centros, así como de una fuerte formación para el profesorado en aspectos pedagógicos del uso de las TIC, puesto que sino dicha política tiende al fracaso en la transformación de las prácticas educativas (Area, 
2011; Campión et al., 2014; Sosa y Valverde, 2014).

\subsection{Innovación Educativa y metodología didáctica con TIC}

Algunos autores afirman que la verdadera integración de las tecnologías en la práctica educativa se producirá definitivamente cuando estas herramientas se caractericen por su invisibilidad y transparencia, ya que se utilizarán de forma habitual y natural en el proceso de enseñanza-aprendizaje (Cabero, 2004; Escontrela y Stojanovi, 2004; Valverde, Garrido, y Fernández, 2010). No obstante, la simple instalación de los equipos informáticos o el uso de éstos para enseñar el mismo contenido, no significa que se esté produciendo la integración de las tecnologías (Protheroe, 2005). En este sentido, se considera que la utilización de herramientas tecnológicas en la práctica docente no garantiza la mejora de los resultados del alumnado (Alonso et al., 2010; Area, 2007). Realmente, lo que incrementa el aprendizaje son otros factores como la manera en que la tecnología es utilizada, "de hecho, los casos en los que se han identificado mejoras en el aprendizaje siempre están relacionados con el uso pedagógicamente innovador de las TIC" (Alonso et al., 2010:56). Por tanto, la implementación de los recursos tecnológicos deben propiciar nuevos modelos de aprendizaje y desarrollar procesos de innovación.

El concepto de "Innovación Educativa" hace referencia al proceso, normalmente deliberado y sistemático que pretende un cambio en la práctica educativa actual (Valverde, 2007). Las autoras nos situamos en la definición de "Innovación Educativa"como un proceso complejo y dinámico que conlleva y origina transformaciones de distinta índole, pudiendo afectar tanto a las actuaciones docentes, como a los aprendizajes de los alumnos, al clima o a los sistemas organizativos de los centros. Partiendo de esta definición se puede afirmar que los indicadores, tales como la dotación de medios en los centros educativos, el número de alumnos por ordenador o el tipo de infraestructuras informáticas, no son criterios a tener en cuenta en la identificación y evaluación de una práctica educativa innovadora (De Pablos, Colás, y Villaciervos, 2010; De Pablos y Jiménez, 2007). Son los indicadores relacionados con los aspectos didácticos, como las características de las actividades o las decisiones metodológicas, los que realmente muestran la innovación y cuál es la integración real de las tecnologías en la práctica educativa (Alonso et al., 2010; Area, 2007b, 2008; Bullock, 2011; Correa, Losada, y Karrera, 2010).

Por tanto, no basta con instalar las tecnologías en los centros, sino que es necesario utilizarlas y hacerlo de manera adecuada pues "la calidad educativa no depende directamente de la tecnología empleada, sino del método de enseñanza bajo el cual se integra el uso de la tecnología, así como de las actividades que realiza el alumno con la misma" (Area, 2007:43). En este sentido, algunos trabajos aluden a aspectos como el trabajo colaborativo, el aprendizaje de naturaleza socioconstructivista, actividades de resolución de problemas o de uso inteligente de la información, como aspectos metodológicos que deben estar presentes en cualquier práctica educativa innovadora TIC para conseguir mejoras en los aprendizajes (Area, 2007b; Cabero, Llorente, y Román, 2007; Correa et al., 2010).

Por tanto, se identifican dos modelos diferenciados en el uso de las TIC según la metodología y tipos de actividades desarrolladas en la práctica de aula, y de los cuales dependerá que el alumno adquiera un aprendizaje más o menos eficaz. Por un lado, el modelo más tradicional y, por otro, el modelo innovador (Escontrela y Stojanovi, 2004; Koster, Kuiper, y Volman, 2012).

Tabla 1. Diferenciación entre modelo tradicional y modelo innovador del uso de las TIC en el aula

\begin{tabular}{|c|c|c|}
\hline & MODELO TRADICIONAL & MODELO INNOVADOR \\
\hline Introducción TIC & Enfoque tecnológico & Enfoque didáctico y pedagógico \\
\hline Enseñanza & Enfoque centrado en el docente & Enfoque centrado en el estudiante \\
\hline Rol docente & Transmitir información al alumno. & Orientador del proceso de aprendizaje \\
\hline Rol del alumno & Aprendizaje pasivo & Aprendizaje activo \\
\hline Plan de estudio & $\begin{array}{l}\text { Iniciativa, control y responsabilidad del } \\
\text { profesor. }\end{array}$ & Iniciativa, control y responsabilidad compartida. \\
\hline Planificación de la clase & Fácil y rápido & Compleja y requieren de más tiempo \\
\hline Actividades & $\begin{array}{l}\text { Aprendizaje descontextualizado y de } \\
\text { tipo memoristico. }\end{array}$ & $\begin{array}{l}\text { Aprendizaje significativas y de tipo exploratorio } \\
\text { e investigación }\end{array}$ \\
\hline Uso de las TIC & $\begin{array}{l}\text { Para ampliar y apoyar el proceso de } \\
\text { enseñanza- aprendizaje }\end{array}$ & $\begin{array}{l}\text { Forma parte del mismo proceso de enseñanza- } \\
\text { aprendizaje }\end{array}$ \\
\hline
\end{tabular}




\begin{tabular}{cll}
\hline $\begin{array}{c}\text { Adaptación al alumno } \\
\text { Resultados de } \\
\text { aprendizaje }\end{array}$ & $\begin{array}{l}\text { Estandarización } \\
\text { Adquisición de conocimiento }\end{array}$ & $\begin{array}{l}\text { Personalización } \\
\text { Adquisición de habilidades }\end{array}$ \\
$\begin{array}{c}\text { Tiempo de aprendizaje } \\
\text { Motivación del alumno }\end{array}$ & $\begin{array}{l}\text { Tiempo constante y } \\
\text { variables } \\
\text { Por el cambio de la herramienta }\end{array}$ & $\begin{array}{l}\text { El estudiante tiene el tiempo que necesita para } \\
\text { alcanzar los resultados deseados } \\
\text { Por la mejora de las actividades }\end{array}$ \\
\hline
\end{tabular}

Fuente: elaboración propia.

\subsection{Análisis de las prácticas educativas con TIC desarrolladas}

Por otra parte, Holhfeld et al (2008) establecen que ha de estudiarse el tipo de práctica educativa para determinar en qué nivel se encuentra el centro en cuanto a la integración de las tecnologías. Para ello considera importante el análisis de la frecuencia con la que los estudiantes y profesores utilizan las herramientas tecnologías en el aula (software, tutoriales, hojas de cálculo o procesadores de texto) y los fines para los que se está utilizando (evaluación, análisis, información, elaboración proyecto curricular). Según la literatura consultada, la práctica educativa desarrollada por los docentes con las TIC, según la finalidad y el tipo de herramienta, que se utilice, se puede dividir en distintos tipos de uso (Area, 2007b, 2008; Inan y Lowther, 2010; Meneses, Fàbregues, RodríguesGómez, y Georgeta, 2012; Ottenbreit-leftwich, Glazewski, Newby, y Ertmer, 2010; Tondeur, Van Braak, y Valcke, 2007):

1. Labores administrativas del docente: a) Para planificar la clase; b) Para comunicarse con la comunidad educativa; c) Para crear materiales didácticos digitales.

2. Para labores relacionadas con el proceso de enseñanza-aprendizaje: a) Para apoyar exposiciones magistrales del profesorado; b) Para consolidar el contenido desarrollado; c) Para ampliar el contenido y adquirir competencias digitales; d) Para enseñar competencias informáticas en el uso de software.

En cuanto a las labores relacionadas con el proceso de enseñanza-aprendizaje, los estudios de investigación señalan que unas prácticas son más habituales que otras, aunque no está claro cuánto tiempo y el tipo de actividades que se dedican al desarrollo de las TIC para la docencia. Aún así, coinciden en que son puntuales, realizadas de manera informal, de forma descontextualizada y sin un valor pedagógico (Area, 2010; Drenoyianni, 2006). Según la bibliografía consultada, las prácticas más habituales son el uso del procesador de texto e Internet, las micro-actividades y explicaciones o exposiciones magistrales con la pizarra digital. Después, en menor proporción, el uso actividades centradas en la creación, elaboración y exposición de contenido por parte del alumnado (Area, 2008, 2010; Area, Sanabria, y Vega, 2013; Domingo y Marquès, 2013; Drenoyianni, 2006; Inan y Lowther, 2010; Ottenbreit-leftwich et al., 2010; Quintero y Hernández, 2005).

La literatura científica también apunta que las prácticas educativas TIC desarrolladas en los centros educativos, mantienen un modelo tradicional. "Los tipos de usos de las TIC hasta el momento no puede y no va a revolucionar la práctica educativa" (Drenoyianni, 2006). Los docentes utilizan la tecnología digital de forma similar a las herramientas no digitales con las que ya están familiarizadas. En pocos casos el uso didáctico de las tecnologías representa cambios metodológicos para contribuir a los procesos de innovación y mejora de la calidad educativa. En la práctica educativa TIC se desarrollan objetivos tecnológicos, más que pedagógicos y didácticos y parece que a los docentes les preocupa más el dominio del medio a qué hacer con ese medio. Además, el protagonismo del uso de las TIC principalmente se centra en el docente, que diseña el proceso de enseñanza-aprendizaje sin tener en cuenta las opiniones, ni las necesidades educativas de los alumnos. Se emplean las TIC fundamentalmente para el trabajo individual del alumnado y en la realización de actividades reproductivas del conocimiento. De manera que el uso de las TIC en el aula está legitimando y reforzando el modelo de educación tradicional (Aguaded, Pérez, y Monescillo, 2010; Area, 2008; L. Fernández et al., 2014; Fernández, 2007; Losada, Karrera, y Correa, 2011; Protheroe, 2005; Quintero y Hernández, 2005; Rubagiza, Were, y Sutherland, 2011; Sanabria, 2006; Sipilä, 2011; Tilve y Álvarez, 2009; Tilve, Gewerc, y Álvarez, 2009).

Así pues, los ordenadores se utilizan principalmente para complementar la práctica educativa más que para cambiar la práctica educativa existente. Los resultados de estudios de investigación apuntan que fundamentalmente se utilizan las TIC con el objetivo de repasar o ampliar el contenido, basados en el "mito de la 
complementariedad" (Tilve y Álvarez, 2009; Tilve et al., 2009). En este sentido, las TIC se utilizan como una herramienta de apoyo a las tareas de transmisión de conocimiento, infravalorando el potencial de las tecnologías ante las herramientas tradicionales como el libro del texto o la pizarra verde que son utilizados en el momento más relevante del proceso de enseñanza-aprendizaje (Area, 2010; Cuadrado, 2008; Quintero y Hernández, 2005; Sigalés, Monimó, y Meneses, 2009; Smeets, 2005).

No obstante, existen otros trabajos de investigación mucho más optimistas que apuntan que las TIC está teniendo un impacto positivo en el cambio de la metodología didáctica desarrollada en los centros educativos. Los resultados indican que, aunque aún el uso de las tecnologías en el aula sigue siendo bastante irregular y discontinuo, cada vez es más frecuente, por lo que consideran que se está produciendo una evolución y transformación en la educación. Según estos estudios se está produciendo un cambio que, poco a poco, permeará en la dinámica institucional. Los docentes están superando el uso tradicional de las TIC en los procesos de enseñanza-aprendizaje, desarrollando un enfoque más centrado en el estudiante (Aguaded et al., 2008; Alonso et al., 2010; Escontrela y Stojanovi, 2004; Hennessy, Ruthven, y Brindley, 2005; Pérez, Aguaded, y Fandos, 2010; M. A. Pérez, Hernando, y Aguaded, 2011; Shahmir, Hamidi, Bagherzadeh, y Salimi, 2011; Wong, Li, Choi, y Lee, 2008).

En cuanto a la labor administrativa del profesorado, según los estudios científicos consultados, son muy pocos los docentes o centros educativos que se lanzan a la creación de materiales didácticos para sus aulas, y aquellos que lo hacen normalmente lo realizan dentro de un convocatoria estatal. El profesorado opta preferentemente por utilizar materiales ya elaborados por otros compañeros de profesión, o provenientes de bancos de recursos institucionales, por lo que suelen ser materiales poco adaptados (Aguaded et al., 2010; Area, 2010; Barrantes, Casas, y Luengo, 2011). El profesorado se puede considerar un agente consumidor más que productor de medio y recursos didácticos (Cabero, 2001).

Por otra parte, en algunos estudios de investigación también queda visible el aumento de uso de las TIC en los centros educativos, aunque en este caso los resultados indican que esta utilización es principalmente de carácter administrativo o burocrático. El profesorado cada vez utilizan las tecnologías con más asiduidad para la organización, evaluación y planificación de actividades, mientras que el uso de las TIC como herramienta didáctica en el proceso de enseñanzaaprendizaje sigue siendo muy bajo. (M. D. Fernández, 2007; Sipilä, 2011).

\section{METODOLOGÍA DE INVESTIGACIÓN}

\subsection{Contexto de la investigación}

Como se ha señalado, en la mayoría de los centros escolares no se percibe una plena integración de las TIC en las aulas y, por tanto, su uso normalizado en el proceso de enseñanzaaprendizaje. No obstante, a pesar de las dificultades de las distintas políticas educativas llevadas a cabo, más concretamente el Proyecto Escuela 2.0, el proceso de introducción de las TIC en el sistema educativo está evolucionando. Cada vez se están produciendo más prácticas educativas que utilizan las tecnologías como una herramienta en el proceso de enseñanza-aprendizaje que pueden constituir pequeños focos de innovación y cambio que poco a poco pueden arraigarse en la dinámica institucional dominante (Alonso et al, 2010).

La intención a lo largo de la investigación ha sido profundizar en los procesos de integración de las TIC en los centros educativos de secundaria, de tal forma que se pueda conocer el estado actual de este proceso y el tipo de prácticas educativas que se están produciendo. Con el proyecto Escuela 2.0 los centros educativos de secundaria de la Comunidad Autónoma de Extremadura han adquirido nuevos recursos tecnológicos, pasando de un modelo de un ordenador por cada dos estudiantes a un modelo $1: 1$, con lo cual es necesario estudiar como este nuevo modelo de integración TIC está influyendo en las prácticas de educativas y como contribuye a la adaptación de los centros a los cambios tecnológicos. Para ello se considera importante analizar las prácticas educativas que se están realizando en torno al modelo 1:1 en distintos institutos de educación secundaria, y que nos puede dar una visión más completa sobre la realidad de escolar en cuanto a la integración de las TIC. En este sentido, los objetivos específicos de la presente investigación son:

1. Comprender el impacto del modelo 1:1 en las prácticas de aula en educación secundaria. 
2. Conocer cuáles son los usos pedagógicos y profesionales de los portátiles por el profesorado y el alumnado.

3. Identificar prácticas educativas innovadoras que puedan favorecer la integración de las tecnologías.

Para la consecución de dichos objetivos era necesario establecer preguntas de investigación y dirigir la atención y el proceso que se llevaría a cabo:

a) ¿Qué impacto tiene el modelo 1:1 sobre la enseñanza?

b) ¿Qué tipo de escenarios y situaciones emergen con la presencia del modelo 1:1 en las aulas de los institutos de secundaria?

c) ¿Pueden ser consideradas las prácticas educativas, en torno al modelo 1:1, que se producen en las aulas como innovación educativa TIC?

d) ¿Qué tendencias educativas parecen consolidarse de cara al futuro?

\subsection{Metodología de investigación: el Estudio de Casos}

Según las características de nuestro problema de investigación y los objetivos planteados, se ha seleccionado una metodología de corte cualitativo por distintas razones. La investigación cualitativa implica una aproximación interpretativa y naturalista del mundo (Stake, 1998:46), lo cual permite estudiar las cosas en su contexto natural, intentando dar sentido o interpretar los fenómenos en función de los significados que las personas le dan (Denzin y Lincoln, 2005:3). Para nuestra investigación este aspecto es muy importante ya que nos interesa la "realidad", tal y como la interpretan los sujetos, respetando el contexto donde dicha "realidad social" es construida

El método con la que se ha llevado a cabo este análisis responde al estudio de casos (Stake, 1998). En los últimos años, el Estudio de Caso ha sido utilizado, como estrategia metodológica de análisis, en muchos trabajos de investigación que tienen como objeto de estudio el uso o incorporación de las TIC en el Sistema Educativo (Vidal, 2006). Ya que esta estrategia metodológica permite "el estudio de la particularidad y de la complejidad de un caso singular, para llegar a comprender su actividad en circunstancias importantes". (Stake, 1998:11-20). Al igual que dichos trabajos, a fin de dar respuesta a los interrogantes se utilizará como unidad de análisis del estudio de caso la totalidad de un centro educativo. Para nuestra investigación, la subunidad de análisis es el aula con un docente que realiza una práctica educativa con TIC. En este sentido, han sido seleccionadas tres experiencias docentes correspondientes a tres Institutos de Educación Secundaria diferentes de la Comunidad Autónoma de Extremadura.

\subsection{Recogida y análisis de datos: La observación}

Para los instrumentos de recogida de información, se ha establecido como conveniente realizar una técnica de observación no participante sobre la actuación del maestro en su aula, mediante notas de campo. La investigación se dirige hacia la identificación y análisis de prácticas educativas con TIC en el marco de la implementación del Proyecto Escuela 2.0. Por tanto, se realizará una observación directa de las prácticas educativas que utilicen el portátil como herramienta didáctica.

El investigador, en este caso, ha desempeñado el papel de investigador ajeno que observa y anota situaciones que le resultan de interés en el momento que está observando, con la menor interferencia posible de su presencia. Se utilizará un sistema narrativo para describir de forma detallada todo lo que acontece durante los 50 minutos, periodo que comprende una clase.

No obstante, la observación cualitativa no es una mera contemplación, como sentarse a ver el mundo y tomar notas, sino que implica adentrarnos en profundidad a situaciones sociales y mantener un papel activo, así como una reflexión permanente (Hernández et al., 2006:587). En este sentido, es importante, que el investigador esté atento a los detalles, sucesos, eventos e interacciones, puesto que de acuerdo con Stake (1998) "las observaciones conducen al investigador a una mejor comprensión del caso". Siguiendo estos preceptos se construyó un guión de registro organizado por temas de interés, porque era necesario acotar los aspectos pertinentes a observar para el caso. Para ello, se adaptaron como base aquellos aspectos que Area (2010) considera destacables en cualquier práctica educativa y que son necesarios considerar para identificar si estamos ante innovaciones educativas de primer orden: «Organización escolar», «Enseñanza, roles e interacción», «Aspectos didácticos» $\mathrm{y}$ «Recursos tecnológicos».

Posteriormente, se realizó la categorización de todas las anotaciones en cada dimensión que establece el guión de registro creado para el análisis de dichos documentos audiovisuales. Finalmente se procedió al análisis de contenido 
cualitativo y la triangulación de todos los datos obtenidos de cada una de las fuentes para llegar a unas conclusiones lo más fiables y cercanas a la realidad.

\section{RESULTADOS}

A continuación se realiza un análisis exhaustivo atendiendo a las siguientes dimensiones: aspectos didácticos, enseñanza, roles e interacción, organización escolar y recursos tecnológicos.

\subsection{Aspectos didácticos}

Como se puede ver en la siguiente tabla de análisis, en las prácticas educativas con TIC se identifican principalmente cuatro tipos de actividades realizadas en el aula.

1. En primer lugar, se desarrolla la exposición del docente. Normalmente, se explica al alumnado algún contenido necesario para la actividad, el modo de desarrollarla, así como los recursos necesarios. En las dos clases en las que tienen PDI, la utilizan para exponer dichos contenidos.

2. En segundo lugar, la búsqueda de páginas web en la red, por indicación del docente, donde encontrarán algunas de las actividades didácticas que van a realizar el alumnado.

3. En tercer lugar, la creación de documentos de presentaciones por parte del alumnado como resultado de un aprendizaje previo, en el que han buscado la información en los recursos web establecidos por la docente.

4. Por último, los alumnos realizan actividades didácticas predeterminadas a las que normalmente se acceden a través de páginas web.
Por tanto, de forma general los objetivos principales de la realización de los ejercicios con TIC es la de consolidar y ampliar conocimientos que previamente se han adquirido en el aula. En general, el alumnado parece no tener demasiados problemas con el desarrollo de las actividades, aunque es necesario señalar que dependiendo de naturaleza de los ejercicios provoca más la demanda de atención del docente para resolver dudas y dificultades.

Adentrándonos en las características de las actividades propuestas se puede observar que solo una de las prácticas educativas exigen una transformación de la información o los contenidos dados. Aunque en las demás prácticas educativas, a través de los materiales didácticos digitales, el docente también pretende llegar a un aprendizaje significativo, en la que las distintas tipologías de ejercicios le sirvan al alumnado consolidar, ampliar y a la vez evaluar los contenidos.

Solo en una de las observaciones se aprecia la creación de productos resultados del aprendizaje, en el que se utiliza la Web para ampliar conocimiento o consultar información. Las otras experiencias didácticas utilizan material didáctico pre-elaborado, a través del cual el alumnado desarrolla ejercicios de lectura, de relacionar, de seleccionar, de elección múltiple, de una forma cerrada, que no favorece la creatividad y el pensamiento crítico.

En todo caso, en ninguna de las prácticas analizadas se han utilizado materiales didácticos tradicionales, ni siquiera en aquella en la que no cuentan con Pizarra Digital Interactiva, puesto que toda la información y recursos necesarios los tienen a través de la web o el docente.

Tabla 2. Aspectos didácticos relativos al uso de los portátiles en el aula

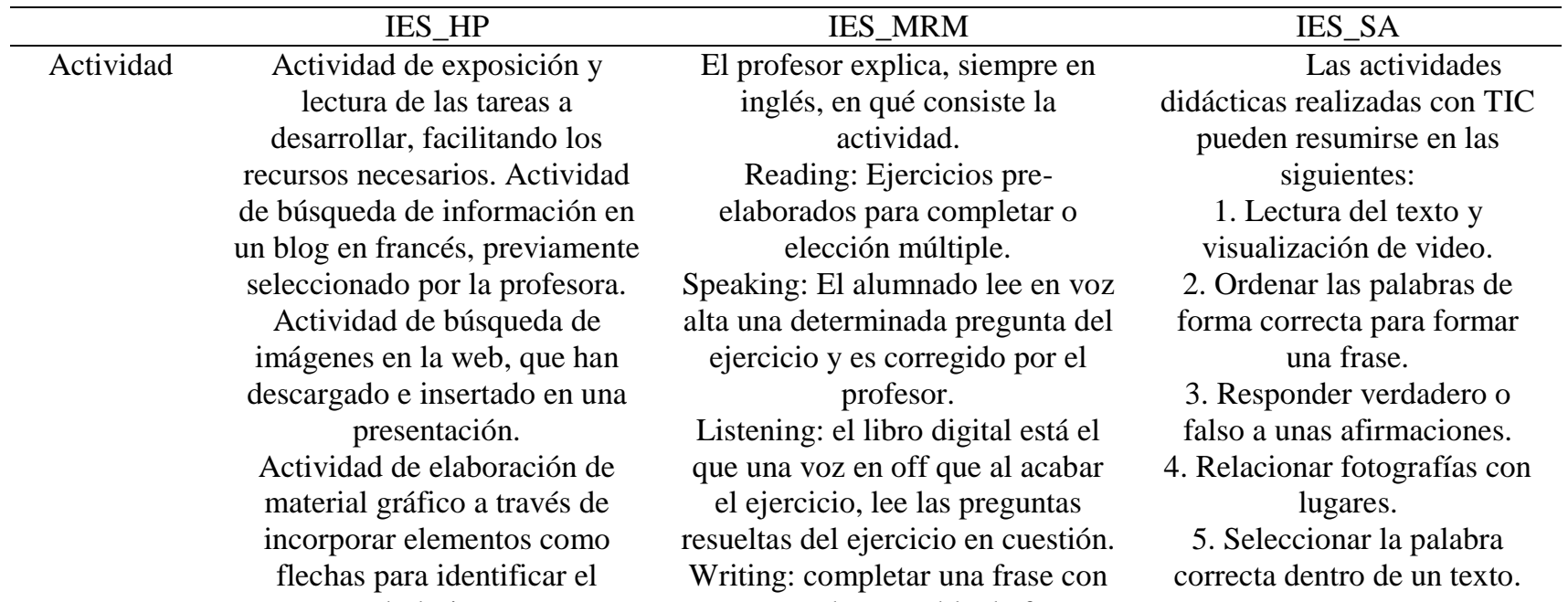


invariables y todos los pronombres.

Material No, uso material tradicional

Recursos Los recursos utilizados por la docente son compartidos en Google Drive.

El alumno utiliza recursos web, como un blog, para extraer la información.

Producto Presentación con Google Docs, documento que comparte con un compañero y la docente.
No, uso material tradicional

Las actividades didácticas vienen propuestas por el libro digital.

No se han producido
No, uso material tradicional Material didáctico interactivo a través de la lectura de un texto y la visualización de un video, propone diversas actividades que el alumno tiene que resolver de forma interactiva. No se han producido.

Fuente: elaboración propia

\subsection{Enseñanza, roles e interacción}

En las tres prácticas educativas las actividades propuestas son meramente individuales en la que el estudiante es el principal protagonista y responsable en su aprendizaje, teniendo este un papel mayoritariamente activo. El estudiante aprende a través de la práctica, inmerso en las actividades propuestas, y realizadas de forma autónoma.

Sin embargo, podría decirse que el alumnado aunque tenga un papel primordialmente activo y realicen los ejercicios con bastante autonomía, gozan de cierta semi-libertad en la realización de éstos, puesto que se imponen en cada actividad unas reglas establecidas para el desarrollo de las tareas, lo que impide el fomento de la creatividad y el pensamiento crítico.

El alumnado aparenta estar más atento y concentrado en la realización de las actividades. Por tanto, se encuentran grupos muy disciplinados. El bullicio que se produce, se corresponde mayormente con la pedida de ayuda al docente, a excepción de algunas situaciones no reseñables. Se puede afirmar que el uso de las TIC en el aula provoca motivación y concentración en el aprendizaje, produciéndose momentos de algarabía, cuando el alumnado tiene dudas y pide la orientación del maestro o la maestra.
Sin embargo, en estas observaciones de aula, no parece ser que se fomente el trabajo colaborativo, ni la comunicación entre el alumnado. Los ejercicios se trabajan de manera individual, aunque se puede observar en algunos momentos del registro de la práctica educativa que los estudiantes reciben ayuda de sus propios compañeros cuando tienen alguna duda o problema y el docente no puede atenderlos.

En cuanto al rol del docente, principalmente tiene la función de guía y orientador en el aprendizaje. Estos solo establecen las indicaciones de cuales son las tareas a realizar y en qué consisten para que el alumnado individualmente intente llevarlas a cabo. Solo en el momento en el que el profesor trata de explicar cómo se deben efectuar estos ejercicios parece darse una enseñanza transmisiva.

No obstante, también el docente cumple funciones de control puesto que, como se puede observar en cada una de las experiencias didácticas, el docente continuamente está controlando el desarrollo de las actividades por parte de los estudiantes para ofrecer la ayuda correspondiente. Además, tiene un rol de líder y autoridad, el cual propicia el buen clima escolar y un buen ritmo de trabajo.

Tabla 3. El rol del alumnado y el profesorado en el uso del portátil

\begin{tabular}{|c|c|c|c|}
\hline & IES_HP & IES_MRM & IES_SA \\
\hline Papel del docente & $\begin{array}{l}\text { - La profesora utiliza un rol de } \\
\text { orientadora del aprendizaje. } \\
\text { - Transmite la información } \\
\text { precisa y materiales didácticos } \\
\text { que aportan los datos e } \\
\text { informaciones necesarias. } \\
\text { - Busca en el alumno una } \\
\text { actitud proactiva, y de } \\
\text { retroalimentación con el } \\
\text { docente. }\end{array}$ & $\begin{array}{l}\text { - El profesor utiliza un rol de } \\
\text { acompañante de las actividades } \\
\text { del libro digital. } \\
\text { - El profesor mantiene una } \\
\text { interacción grupal y individual: } \\
\text { pregunta, reclama la atención, } \\
\text { refuerza. } \\
\text { - Nunca se sienta y está } \\
\text { observando constantemente lo } \\
\text { que ocurre en el aula. }\end{array}$ & $\begin{array}{c}\text { - El papel desarrollado por } \\
\text { la docente, es meramente } \\
\text { orientador. } \\
\text { - La docente propone la } \\
\text { actividad y solo interviene } \\
\text { para señalar los recursos. } \\
\text { - Aclara algunas dudas } \\
\text { sobre como resolver la } \\
\text { actividad } \\
\text { Traduce algunas palabras }\end{array}$ \\
\hline
\end{tabular}




\begin{tabular}{|c|c|c|c|}
\hline & $\begin{array}{l}\text { - Busca y preparación del } \\
\text { material didáctico. } \\
\text { - Muestra atención } \\
\text { personalizada y grupal: } \\
\text { pregunta, reclama la atención, } \\
\text { refuerza, responde, observa } \\
\text { entre los pupitres. } \\
\text { - Nunca se sienta y está } \\
\text { observando constantemente lo } \\
\text { que ocurre en el aula. } \\
\text { - Mantiene clara su autoridad } \\
\text { sobre el grupo, pero sabe } \\
\text { establecer un clima de aula muy } \\
\text { positivo. }\end{array}$ & $\begin{array}{l}\text { - Mantiene clara su autoridad } \\
\text { sobre el grupo, pero sabe } \\
\text { establecer un clima de aula } \\
\text { agradable. } \\
\text { - Aclara y refuerza contenidos y } \\
\text { conceptos. }\end{array}$ & $\begin{array}{c}\text { que el alumnado no } \\
\text { entienda. } \\
\text { - La profesora también } \\
\text { cumple el rol de control del } \\
\text { aula. La docente controla } \\
\text { las pantallas de los } \\
\text { portátiles para valorar el } \\
\text { desarrollo de las } \\
\text { actividades e identificar } \\
\text { alguna dificultad. }\end{array}$ \\
\hline Papel del alumno & $\begin{array}{l}\text { Los estudiantes hablan entre } \\
\text { ellos y preguntan a la profesora } \\
\text { en cualquier momento. } \\
\text { Reclaman su atención } \\
\text { levantando la mano. } \\
\text { El alumnado se compromete en } \\
\text { la tarea y sigue las indicaciones } \\
\text { de la profesora. }\end{array}$ & $\begin{array}{c}\text { Se limitan a responder a los } \\
\text { ejercicios planteados, oralmente } \\
\text { y por escrito. } \\
\text { La clase se involucra por } \\
\text { participaciones individuales } \\
\text { solicitadas por el profesor. } \\
\text { El alumnado habla poco, } \\
\text { absorvidos por los ejercicios del } \\
\text { libro digital, y las indicaciones y } \\
\text { explicaciones. } \\
\text { Los estudiantes levantan la } \\
\text { mano cuando el profesor pide } \\
\text { voluntarios para leer. } \\
\text { Algunos reclaman su } \\
\text { atención levantando la mano. }\end{array}$ & $\begin{array}{l}\text { Se percibe que el alumnado } \\
\text { se compromete en la tarea y } \\
\text { de forma disciplinada } \\
\text { realizan la tarea con } \\
\text { atención. }\end{array}$ \\
\hline Colaboración & $\begin{array}{l}\text { La actividad es individual, } \\
\text { aunque los alumnos interactúan } \\
\text { para compartir información. }\end{array}$ & $\begin{array}{l}\text { La actividad es individual, } \\
\text { aunque los alumnos interactúan } \\
\text { para compartir información. }\end{array}$ & $\begin{array}{l}\text { La actividad es individual y } \\
\text { la realización está siendo } \\
\text { totalmente autónoma por } \\
\text { parte de los estudiantes. }\end{array}$ \\
\hline
\end{tabular}

Fuente: elaboración propia

\subsection{Organización escolar}

En las prácticas de aula que se han analizado, las tecnologías de la Información y Comunicación son el principal protagonista en el aprendizaje. Durante todo el desarrollo de la clase, el alumno ha tenido una constante interacción con la tecnología.

En cuanto a la organización y distribución de los puestos y mesas, se realiza de modo tradicional, a través de filas contiguas, unas detrás de otras, y todas de cara a la pizarra y el puesto del docente. Aunque normalmente, las mesas son agrupadas por pareja, no responde a objetivos de fomento del trabajo en equipo o interacción del alumnado, puesto que las actividades son meramente individuales.

Esta distribución, aunque las sillas puedan trasladarse a otros puestos, las mesas están fijas en el suelo. Las mesas tienen un dispositivo para conectar el portátil, por lo que no pueden moverse. Por este motivo se dificulta la realización de actividades grupales y trabajos en equipo, que pudieran favorecer la colaboración. Además, no se percibe que ninguno de los docentes realice gestión alguna respecto a la distribución tanto de los equipos informáticos como de las mesas, por lo que se considera que están previamente asignados desde comienzo de curso, y los alumnos utilizan con asiduidad estos recursos tecnológicos en el aula.

En cuanto a la distribución del tiempo de la clase, el docente dedica la mayor parte del tiempo principalmente a tres tareas: dar las instrucciones de las tareas, supervisar el trabajo del alumnado, y resolver las dudas. Con esto se corrobora una vez más que el uso didáctico de las TIC facilita el cambio de rol del profesorado, cumpliendo solo la función de orientador y guía en el aprendizaje y dejando que el protagonista sea el alumnado. Pues, prácticamente, la totalidad del tiempo de la sesión el alumno la ocupa realizando la tarea didáctica encomendada por el docente. 
Tabla 4. Aspectos organizativos en el uso de los portátiles en el aula

\begin{tabular}{|c|c|c|c|}
\hline & IES_HP & IES_MRM & IES_SA \\
\hline $\begin{array}{l}\text { Organización del } \\
\text { aula }\end{array}$ & $\begin{array}{l}3 \text { filas de } 6 \text { mesas con dos } \\
\text { puestos cada una. } \\
\text { En el aula hay } 22 \text { alumnos. } \\
\text { Una mesa para el profesor con } \\
\text { un ordenador de sobremesa y } \\
\text { conectado a altavoces y a un } \\
\text { videoproyector. } \\
\text { Las mesas no pueden ser } \\
\text { movidas. } \\
\text { No existe una indicación } \\
\text { expresa sobre el modo de } \\
\text { agruparse. Cada alumno ha } \\
\text { ocupado el que parece ser su } \\
\text { puesto habitual en el aula. } \\
\text { Si es necesario se agrupan en } \\
\text { pareja para compartir portátil. }\end{array}$ & $\begin{array}{l}5 \text { filas con tres mesas de dos } \\
\text { puestos cada una. } \\
\text { En el frente del aula, a la } \\
\text { izquierda está la mesa del } \\
\text { profesor con un ordenador de } \\
\text { sobremesa, la PDI detrás, y a } \\
\text { su derecha una pizarra de } \\
\text { rotuladores. } \\
\text { La clase la conforman } 27 \\
\text { alumnos. } \\
\text { Los puestos son movibles. } \\
\text { No existe una indicación } \\
\text { expresa sobre el modo de } \\
\text { agruparse. Cada alumno ha } \\
\text { ocupado el que parece ser su } \\
\text { puesto habitual en el aula. }\end{array}$ & $\begin{array}{c}\text { Se distribuyen en tres filas } \\
\text { con cinco mesas dobles } \\
\text { cada una, albergando a } 30 \\
\text { alumnos. } \\
\text { Las mesas son de movilidad } \\
\text { reducida, debido a un } \\
\text { dispositivo de conexión que } \\
\text { tienen. } \\
\text { Mesa para el profesor la } \\
\text { cual está equipada con un } \\
\text { equipo informático de } \\
\text { sobremesa conectados a } \\
\text { altavoces. } \\
\text { No existe una indicación } \\
\text { expresa sobre el modo de } \\
\text { agruparse. Cada alumno ha } \\
\text { ocupado el que parece ser } \\
\text { su puesto habitual en el } \\
\text { aula. } \\
\text { Si es necesario se agrupan } \\
\text { en pareja para compartir } \\
\text { portátil. }\end{array}$ \\
\hline $\begin{array}{l}\text { Distribución del } \\
\text { tiempo }\end{array}$ & $\begin{array}{l}\text { La mayor parte del tiempo de la } \\
\text { sesión se ocupa en la actividad } \\
\text { de los alumnos. } \\
\text { La profesora intenta marcar un } \\
\text { ritmo más rápido en las } \\
\text { actividades que realizan los } \\
\text { alumnos. } \\
\text { El tiempo que la profesora } \\
\text { dedica a la explicación de } \\
\text { contenidos o de tareas es } \\
\text { reducido. } \\
\text { Interrumpe de forma breve la } \\
\text { actividad de los estudiantes para } \\
\text { aclarar dudas o para presentar } \\
\text { nueva información. }\end{array}$ & $\begin{array}{l}\text { La mayor parte del tiempo de } \\
\text { la sesión se ocupa en la } \\
\text { actividad de los alumnos. } \\
\text { El ritmo es un ritmo relajado, } \\
\text { marcado por el profesor y su } \\
\text { interacción con los estudiantes. } \\
\text { Solo en el último ejercicio se } \\
\text { marcó un límite temporal a } \\
\text { través de un icono de } \\
\text { despertador proyectado. }\end{array}$ & $\begin{array}{c}\text { Sin contar el tiempo } \\
\text { dedicado a distribuir los } \\
\text { portátiles entre los alumnos, } \\
\text { se dedica todo el desarrollo } \\
\text { de la clase a la resolución } \\
\text { de las actividades. } \\
\text { En el transcurso de la } \\
\text { realización de las } \\
\text { actividades de los alumnos, } \\
\text { la profesora interviene en } \\
\text { varias ocasiones para } \\
\text { explicar o ayudar a } \\
\text { entender una actividad. }\end{array}$ \\
\hline Frecuencia de uso & $\begin{array}{c}\text { Toda la sesión de aula se han } \\
\text { utilizado TIC. }\end{array}$ & $\begin{array}{c}\text { Toda la sesión de aula se han } \\
\text { utilizado TIC. }\end{array}$ & $\begin{array}{c}\text { Toda la sesión de aula se } \\
\text { han utilizado TIC. }\end{array}$ \\
\hline
\end{tabular}

Fuente: elaboración propia

\subsection{Recursos tecnológicos}

Las experiencias educativas grabadas se han realizado en la clase habitual del grupo de alumno. El equipamiento tecnológico del aula responde a las características propias de un Aula Digitales del Siglo XXI. Así pues, cada estudiante cuenta con un ordenador portátil, y de conexión a Internet. También cuenta con una Pizarra Digital Interactiva, a la cual siempre le acompaña la Pizarra Verde Tradicional o Pizarra Blanca Especializada. En una de las aulas, además cuentan con armarios en los que se guardan los equipos informáticos, junto a su cableado, bajo llave, lo que provoca perder una media de 5 minutos en cada clase para repartir y recoger los ordenadores. No obstante, dos de los Institutos permiten llevar los ordenadores portátiles a casa, aunque obligan a firmar un contrato de compromiso y cuidado al alumnado para el correcto funcionamiento de los portátiles.

El sistema operativo con el que cuenta los equipos informáticos es GnuLinex, que junto a los software que le acompañan suponen la transmisión indirecta de valores de colaboración y solidaridad, entre otros, debido a su condición de software libre. 
En cuando a los aspectos técnicos no se han producido ningún problema significativo. En todo caso, si estos se han producido han sido resueltos por el docente, el cual ha solucionado el problema en el ordenador o se han agrupado los estudiantes por propia iniciativa para la realización de la actividad.

Tabla 5. Accesibilidad y disponibilidad de recursos tecnológicos

\begin{tabular}{|c|c|c|c|}
\hline & IES_HP & IES_MRM & IES_SA \\
\hline Infraestructuras & $\begin{array}{c}\text { Ordenadores Portátiles para } \\
\text { cada uno de los alumnos. Una } \\
\text { Pizarra Digital Interactiva, y } \\
\text { una pizarra Verde } \\
\text { Tradicional. } \\
\text { Los portátiles pueden ser } \\
\text { llevados a casa, pero el } \\
\text { alumno tiene la obligación de } \\
\text { tener cargada la batería. } \\
\text { En el aula se conecta a } \\
\text { Internet por cable }\end{array}$ & $\begin{array}{l}\text { Ordenadores Portátiles para } \\
\text { cada uno de los alumnos, que } \\
\text { los alumnos pueden llevar a } \\
\text { casa. } \\
\text { En el aula tienen Internet por } \\
\text { cable y wifi. }\end{array}$ & $\begin{array}{c}\text { Cada uno de ellos alumnos } \\
\text { disponen de un ordenador portátil. } \\
\text { No se permite llevar los } \\
\text { ordenadores a casa. Éstos se } \\
\text { guardan en un armario bajo llave } \\
\text { durante las materias en el que el } \\
\text { alumnado no los utiliza. } \\
\text { No cuenta con PDI, ni Pizarra } \\
\text { Blanca Especializada. }\end{array}$ \\
\hline Software & $\begin{array}{l}\text { Tienen instalado } \\
\text { como sistema operativo } \\
\text { gnuLinex. En la sesión se } \\
\text { utiliza el siguiente software: } \\
\text { Aula Linex, Google Docs } \\
\text { (presentaciones) Mozilla } \\
\text { Firefox (navegador) Google } \\
\text { Drive. }\end{array}$ & $\begin{array}{c}\text { Tienen instalado como } \\
\text { sistema operativo gnuLinex. } \\
\text { En la sesión se utiliza el } \\
\text { navegador (Google Chrome) } \\
\text { para acceder la plataforma } \\
\text { educativa. }\end{array}$ & $\begin{array}{c}\text { Tienen instalado como sistema } \\
\text { operativo gnuLinex. } \\
\text { Página web del Centro de } \\
\text { Profesores y Recursos de Mérida } \\
\text { (http://cprmerida.wikispaces.com). }\end{array}$ \\
\hline $\begin{array}{l}\text { Problema } \\
\text { Técnico }\end{array}$ & $\begin{array}{l}\text { Durante la sesión se } \\
\text { reubicaron en funciones d } \\
\text { ellas necesidades para } \\
\text { compartir ordenador, puesto } \\
\text { que alguno no lo han traído. }\end{array}$ & & $\begin{array}{c}\text { Los alumnos se agrupan en pareja } \\
\text { si tienen problemas técnicos con } \\
\text { su portátil. }\end{array}$ \\
\hline
\end{tabular}

Fuente: elaboración propia

\section{CONCLUSIONES}

A tenor de la política de implantación de un "ordenador por niño" o modelo 1:1, se observa que aún las experiencias didácticas con TIC llevadas a cabo en los centros educativos de secundaria aún son esporádicas y se utilizan para complementar los contenidos didácticos desarrollados con anterioridad (Area, 2010; Sigalés, Monimó, y Meneses, 2009; Tilve y Álvarez, 2009). Aunque se puede afirmar que el hecho de disponer de ordenador portátil dentro del aula, favorece que el docente utilice este con más asiduidad.

En el presente trabajo se han identificado y analizado tres prácticas educativas desarrolladas de secundaria en el marco de implementación del Proyecto Escuela 2.0. Al igual que otros estudios de investigación, en dichas experiencias educativas, no se observa una ruptura total con la escuela tradicional (Fernández et al., 2014). En estas prácticas educativas, los docentes no emplean las tecnologías como herramienta sino como fin último. Pese a la implantación de los nuevos ordenadores portátiles y acceso a la red, tan solo una práctica educativa observada favorece la búsqueda y tratamiento de la información. Siguen basando su metodología didáctica en materiales interactivos pre-elaborados (Area, 2010; Barrantes, Casas, y Luengo, 2011) y no se potencia su capacidad de decisión y selección de actividades, proyectos y temas de interés (Fernández et al., 2014).

En cuanto al aprendizaje es principalmente individual, pues no se percibe que se haya realizado trabajo en pareja o en grupo, ni que el profesorado observado no haya fomentado la comunicación entre los estudiantes. No obstante, se puede afirmar que el alumnado se ayuda y solidarizan unos con los otros, aunque no es concluyente que esta solidaridad se dé debido al uso didáctico del ordenador.

La introducción de los portátiles en las aulas también ha influido de manera significativa en la distribución de las mesas y puestos de los alumnos, ya que debido a las necesidades de conexión las mesas deben estar pegadas al suelo y estableciendo estas en modelos tradicionales de organización, en la que las mesas se disponen en filas contigua, unas de tras de otras. Por lo que también en este 
sentido, la organización del aula responde a un modelo tradicional que limita las posibilidades de llevar a cabo trabajo en equipo y la colaboración entre el alumnado.

Por otra parte, se puede concluir que el uso de las TIC como herramienta didáctica, promueve el cambio de rol docente, ostentando principalmente éste, funciones de orientador y guía, mientras el alumnado es activo y autónomo en su propio proceso de enseñanza-aprendizaje. Así mismo, se puede afirmar que el uso de las TIC favorece la motivación del alumnado hacia el aprendizaje. Como se ha podido comprobar, todos los estudiantes muestran un interés en el contenido debido a lo atractivo de la herramienta y la actividad TIC.

Por tanto, las experiencias educativas a través del uso del ordenador portátil que han sido analizadas, responde a un modelo tradicional de enseñanza-aprendizaje y poco innovador (Koster, Kuiper, y Volman, 2012). Así pues, se corrobora que la presencia de dispositivos TIC y el acceso universal a la tecnología, en los centros no cambia necesariamente las estrategias de enseñanza, ni provoca por sí mismas la innovación y transformación educativa (Valiente, 2011). Es necesario, por tanto, instar al profesorado a utilizar las tecnologías con metodologías didácticas más innovadoras, basados en un aprendizaje socioconstructivista y que fomenten la colaboración (Area, 2007b; Cabero, Llorente, y Román, 2007; Correa et al., 2010). Sin embargo, las características intrínsecas de las herramientas tecnológicas promueve en sí mismas la motivación del estudiante y favorece el desarrollo de aprendizajes más activo y centrados en el estudiante.

\section{REFERENCIAS}

Aguaded, J. I., Pérez, M. amor, y Monescillo, M. (2010). Hacia una integración curricular de las TIC en los centros educativos andaluces de primaria y secundaria. Bordón, 4, 7-23.

Aguaded, J. I., Tirado, R., y Cabero, J. (2008). Los centros TIC en Andalucía, españa: un modelo de implementación del profesorado en la integración curricular de la tecnología. Sociotam, 18(2), 171-199.

Alonso, C., Casablancas, S., Domingo, L., Guitert, M., Moltó, Ó., Sánchez, J.-A., y Sancho, J. (2010). De las propuestas de la Administración a las prácticas del aula. Revista de educación, 352, 53-76.

Area, M. (2007). Algunos principios para el desarrollo de "buenas prácticas" pedagógicas con las TIC en el aula. Comunicación y Pedagógía: Nuevas tecnologías y recursos didácticos, 222, 42-47.
Area, M. (2008). Innovación Pedagógica con TIC y el desarrollo de las competencias informacionales y digitales. Investigación en la escuela, 64, 5-18.

Area, M. (2010). El proceso de integración y uso pedagógico de las TIC en los centros educativos. Un estudio de casos. Revista de educación, 352, 77-98.

Area, M. (2011). Los efectos del modelo 1:1 en el cambio educativo en las escuelas. Evidencias y desafíos para las políticas iberoamericanas. Revista Iberoamericana de Educación, 56, 49-74.

Area, M., Alonso, C., Correa, J. M., Moral, M. E., De-PablosPons, J., Paredes, J., Valverde, J. (2014). Las políticas educativas TIC en España después del Programa Escuela 2.0: las tendencias que emergen. RELATEC: Revista Latinoamericana de Tecnología Educativa, 13(2), 11-33.

Area, M., Sanabria, A., y Vega, A. M. (2013). Las políticas educativas TIC (Escuela 2.0) en las Comunidades Autónomas de España desde la visión del profesorado. Revista científica de Tecnología Educativa, 3(1), 7488.

Barrantes, G., Casas, L. M., y Luengo, R. (2011). Obstáculos percibidos para la integración d elas TIC por los profesores de infantil y primaria en Extremadura. Pixel-bit. Revista de Medios y Educación, 39(39), 8394.

Bullock, S. M. (2011). Teaching 2.0: (re)learning to teach online. Interactive Technology and Smart Education, $8(2)$, 94-105. http://doi.org/10.1108/17415651111141812

Cabero, J. (2001). Tecnología Educativa. Diseño y utilización de medios en la enseñanza. Barcelona: Paidós.

Cabero, J. (2004). Cambios organizativos y administrativos para incorporación de las TICs a la formación. Medidas a adoptar. Edutec: Revista electrónica de tecnología educativa, 18, 1-31.

Cabero, J., Llorente, M. C., y Román, P. (2007). La tecnología cambió los escenarios: el efecto Pigmalión se hizo realidad. Comunicar, 28, 167-175.

Campión, R. S., Navaridas, F., y Repáraz, C. (2014). La escuela 2.0: reflexiones en torno a su eficacia en los centros educativos de La Rioja. Educación XX1: Revista de La Facultad de Educación, 17(1), 243-270.

Correa, J. M., Losada, D., y Karrera, I. (2010). ICT policies in schools and their effect on pedagogical innovation in the Spain: the Amara Berri Basque School case study. Procedia Social and Behavioral Sciences, 9, 44-47. http://doi.org/http://dx.doi.org/10.1016/j.sbspro.2010.1 2.113

Cuadrado, I. (2008). Estado de necesidades, desarrollo y evaluación en formación del profesorado en TICs: El caso de la comunidad Autónoma de Extremadura. Avances en Supervisión Educativa: Revista de la Asociación de Inspectores de Educación en España, 8 , $1-13$.

De Pablos, P., Colás, P., y Villaciervos, P. (2010). Políticas educativas, buenas prácticas y TIC en la Comunidad Autónoma Andaluza. Revista teoría de la educación. Educación y cultura en la Sociedad de la Información, 11(1), 180-202.

De Pablos, P., y Jiménez, R. (2007). Buenas Prácticas con TIC apoyadas en las Políticas educativas: claves conceptuales y derivaciones para la formación en competencias ECTS. RELATEC: Revista Latinoamericana de Tecnología Educativa, 6(2), 1528. 
Denzin, N. K., y Lincoln, Y. S. (2005). The Sage Handbook of Qualitative Research. Londres: SAGE Publications.

Domingo, M., y Marquès, P. (2013). Práctica docente en aulas 2.0 de centros de educación primaria y secundaria de España. Pixel-bit. Revista de Medios y Educación, 42, $115-128$.

Drenoyianni, H. (2006). Reconsidering change and ICT: Perspectives of a human and democratic education. Education and Information Technologies, 11, 401413. http://doi.org/10.1007/s10639-006-9005-5

Escontrela, R., y Stojanovi, L. (2004). La integración de las TIC en la educación: apuntes para un modelo pedagógico pertinente. Revista de pedagogía, 25(74).

Fernández, L., Correa, J. M., y Ochoa-Aizpurua, B. (2013). Eskola 2.0: Un curso completado. Tiempo de evaluación. Fuentes: Revista de La Facultad de Ciencias de La Educación, (13), 239-262.

Fernández, L., Losada, D., y Correa, J. M. (2014). Análisis intercasos de prácticas TIC en las aulas de Educación Primaria con un modelo 1:1. Profesorado: Revista de Curriculum Y Formación Del Profesorado, 18(3), 2740.

Fernández, M. D. (2007). ¿Contribuyen las TIC a hacer de los profesores mejores profesionales?: ¿Qué dicen los directivos escolares gallegos? Pixel-bit. Revista de Medios y Educación, 30, 5-15.

Hennessy, S., Ruthven, K., y Brindley, S. (2005). Teacher perspectives on integranting ICT into subject teaching: commitment, constrainsts, caution, and change. Journal of Curriculum Studies, 37(2), 155-192. http://doi.org/10.1080/0022027032000276961

Hernández, R., Fernández-Collado, C., y Baptista, P. (2006). Metodología de la investigación. Mexico: McGraw Hill.

Inan, F., y Lowther, D. (2010). Factors affecting technology integration in K-12 classrooms: a path model. Education Tech Research Dev, 58, 137-154. http://doi.org/0.1007/s11423-009-9132-y

Koster, S., Kuiper, E., y Volman, M. (2012). Concept-guided development of ICT use in "traditional" and "innovative" primary schools: what types of ICT use do schools develop? Journal of Computer Assisted Learning, 28(5), 454-464. http://doi.org/10.1111/j.1365-2729.2011.00452.x

Lagos, M. (2011). Estado de las experiencias 1 a 1 en iberoamérica. Revista Iberoamericana de Educación, 56, 75-94.

Losada, D., Karrera, I., y Correa, J. M. (2011). Políticas sobre la integración de las TIC en la escuela de la Comunidad Autonoma del País Vasco. RELATEC: Revista Latinoamericana de Tecnología Educativa, 10(1), 21-35.

Meneses, J., Fàbregues, S., Rodrígues-Gómez, D., y Georgeta, I. (2012). Internet in teachers' professional practice outside the classroom: Examining supportive and management uses in primary and secondary school. Computers y Education, 59(3), 915-924. http://doi.org/http://dx.doi.org/10.1016/j.compedu.201 2.04.011

Murillo, J. L. (2010). Programas Escuela 2.0 y Pizarra Digital: un paradigma de mercantilización del sistema educativo a través de las TICs. Revista Electrónica Interuniversitaria de Formación Del Profesorado, 13(2), 65-78.

Ottenbreit-leftwich, A., Glazewski, K., Newby, T., y Ertmer, P. A. (2010). Teacher value beliefs associated with using technology: Addressing professional and student needs. Computers y Education, 55, 1321-1335. http://doi.org/10.1016/j.compedu.2010.06.002

Pérez, A. (2011). Escuela 2.0. ¿Por qué en este momento? Retrieved

from http://www.ite.educacion.es/es/escuela-20

Pérez, M. A., Aguaded, J. I., y Fandos, I. (2010). Percepciones, retos y demandas de los directores y coordinadores de los centros TIC andaluces. Siglo $X X I, 28(1)$.

Pérez, M. A., Hernando, Á., y Aguaded, J. I. (2011). La integración de las TIC en los centros educativos: percepciones de los coordinadores y directores. Estudios Pedagogicos, 307(2), 197-211.

Protheroe, N. (2005). Technology and student achievement. National Association of Elementary School Principals, 82(2), 46-48.

Quintero, A., y Hernández, A. (2005). El profesor ante el reto de integrar las TIC en los procesos de enseñanza. Enseñanza y Teaching: Revista interuniversitaria de didáctica, 23, 305-321.

Rubagiza, J., Were, E., y Sutherland, R. (2011). Introducing ICT into schools in Rwanda: Educational challenges and opportunities. International journal of Educational Development, 31(1), 37-43. http://doi.org/http://dx.doi.org/10.1016/j.ijedudev.2010 .06 .004

Sanabria, A. (2006). Las TIC en el sistema escolar de Canarias: Los programas institucionales de innovación educativa para la integración curricular de las Tecnologías de la Información y Comunicación. RELATEC: Revista Latinoamericana de Tecnología Educativa, 5(2), 191-202.

Shahmir, S., Hamidi, F., Bagherzadeh, Z., y Salimi, L. (2011). Role of ICT in the curriculum educational system. Procedia Computer Science, 3, 623-626. http://doi.org/http://dx.doi.org/10.1016/j.procs.2010.1 2.104

Sigalés, C., Monimó, J., y Meneses, J. (2009). TIC e innovación en la educación escolar española. Estado y perspectivas. Cuadernos de Comunicación $e$ Innovación, 78 .

Sipilä, K. (2011). No pain, no gain? Teachers implementing ICT in instruction. Interactive Technology and Smart Education, 8(1), 39-51. http://doi.org/10.1108/17415651111125504

Smeets, E. (2005). Does ICT contribute to powerful learning environments in primary education? Computers $y$ Education, 44, 343-355. http://doi.org/10.1016/j.compedu.2004.04.003

Sosa, M. J., y Valverde, J. (2014). Centros educativos ecompetentes en el modelo 1:1. el papel del equipo directivo, la coordinación TIC y el clima organizativo. Revista de Currículum Y Formación Del Profesorado, 18(3), 41-62.

Stake, R. E. (1998). Investigación con estudio de casos. Madrid: Morata.

Tilve, M. D., y Álvarez, Q. (2009). Un estudio de caso sobre un proyecto de innovación con TIC en un centro educativo de Galicia: ¿Acción o reflexión? Bordón, 61(1), 95-108.

Tilve, M. D., Gewerc, A., y Álvarez, Q. (2009). Proyectos de innovación curricular mediados por TIC: Un estudio de caso. RELATEC: Revista Latinoamericana de Tecnología Educativa, 8(1), 6581. 
Tondeur, J., Van Braak, J., y Valcke, M. (2007). Towards a typology of computer use in primary education. Journal of Computer Assisted Learning, 23, 197-206.

Vaillant, D., Rodríguez, E., y Bernasconi, G. (2015). En qué cambian las prácticas de enseñanza de la matemática en un "modelo 1:1" a escala nacional. Revista Complutense de Educación, 26(2), 295-313. http://doi.org/10.5209/rev_RCED.2015.v26.n2.43059

Valiente, O. (2011). Los modelos 1:1 en Educación. Prácticas internacionales, evidencia comparada e implicaciones políticas. Revista Iberoamericana de Educación, (56), $113-134$.

Valverde, J. (2007). El software libre y las "buenas prácticas" educativas con TIC. Comunicación Y Pedagógía: Nuevas Tecnologías Y Recursos Didácticos, 222(2), $48-55$.

Valverde, J., Garrido, M. C., y Fernández, M. R. (2010). Enseñar y aprender con Tecnologías: Un modelo teórico para las buenas prácticas con TIC. Revista teoría de la educación. Educación y cultura en la Sociedad de la Información, 11(3), 203-229.

Vidal, M. del P. (2006). Investigación de las TIC en la educación. RELATEC: Revista Latinoamericana de Tecnología Educativa, 5, 539-552.

Wong, E. M. L., Li, S. S. C., Choi, T., y Lee, T. (2008). Insights into Innovative Classroom Practices with ICT: Identifying the Impetus for Change. Educational Technology y Society, 11(1), 248-265. 